\title{
Peri-operative, functional and early oncologic outcomes of salvage robotic- assisted radical prostatectomy after high- intensity focused ultrasound partial ablation
}

\author{
James E. Thompson ${ }^{1,2^{*}}$ (D), Ashwin N. Sridhar ${ }^{1,3}$, Greg Shaw ${ }^{1,3}$, Prabhakar Rajan ${ }^{1,4}$, Anna Mohammed ${ }^{1}$,
}

Timothy P. Briggs ${ }^{1}$, Senthil Nathan ${ }^{1,3}$, John D. Kelly ${ }^{1,3}$ and Prasanna Sooriakumaran ${ }^{1,3,5}$

\begin{abstract}
Background: Partial ablation of the prostate using high-intensity focussed ultrasound (HIFU-PA) is a treatment option for localised prostate cancer. When local recurrence occurs, salvage robot-assisted radical prostatectomy is a treatment option for selected patients, but there is a paucity of data on the peri-operative safety, functional and oncologic outcomes of sRARP.

The objective of this study was therefore to describe peri-operative safety, functional and early oncologic outcomes following salvage robot-assisted radical prostatectomy (sRARP) for local recurrence after HIFU-PA.

Methods: Retrospective analysis of a prospective database of 53 consecutive men who underwent sRARP after HIFU-PA from 2012 to 2018. Continence and erectile-function were reported pre-HIFU, pre-sRARP, 3-months postsRARP and 12-months post-sRARP. Complications, PSMs and need for subsequent ADT/radiotherapy were assessed.

Results: 45 men were suitable for inclusion and had sufficient data for analyses. Median duration from HIFU to sRARP was 30.0 months and median follow-up post-sRARP was 17.7 months. Median age, PSA and ISUP group were $63.0 \mathrm{yrs} ., 7.2 \mathrm{ng} / \mathrm{mL}$ and $2 ; 88.9 \%$ were cT2.

Median operative-console time, blood loss and hospital stay were $140 \mathrm{~min}, 200 \mathrm{ml}$ and 1 day respectively. Clavien-Dindo grade 1, 2 and 3 complications < 90 days occurred in 8.9, 6.7 and 2.2\%; late (>90d) complications occurred in 13.2\%. At sRARP pathology, ISUP 3-5 occurred in 51.1\%, pT3a/b in 64.5\%, and PSMs in 44.4\% (37.5\% for pT2, 48.3\% for pT3). Of men with > 3-months follow-up after sRARP, 26.3\% underwent adjuvant radiotherapy/ADT for residual disease or adverse pathologic features; $5.3 \%$ experienced BCR requiring salvage ADT/radiotherapy. Freedom from ADT/radiotherapy was $66.7 \%$ at 12 -months.

Pad-free rates were 100\% pre-HIFU, 95.3\% post-HIFU, 29.4\% 3-months post-sRARP, and 65.5\% 12-months post-sRARP. (Continued on next page)
\end{abstract}

\footnotetext{
* Correspondence: drjethompson@gmail.com

'Department of Uro-oncology, University College London Hospital, London W1G 8PH, UK

${ }^{2}$ Faculty of Medicine, University of New South Wales, Kensington, Australia Full list of author information is available at the end of the article
}

C C The Author(s). 2020 Open Access This article is licensed under a Creative Commons Attribution 4.0 International License, which permits use, sharing, adaptation, distribution and reproduction in any medium or format, as long as you give appropriate credit to the original author(s) and the source, provide a link to the Creative Commons licence, and indicate if changes were made. The images or other third party material in this article are included in the article's Creative Commons licence, unless indicated otherwise in a credit line to the material. If material is not included in the article's Creative Commons licence and your intended use is not permitted by statutory regulation or exceeds the permitted use, you will need to obtain permission directly from the copyright holder. To view a copy of this licence, visit http://creativecommons.org/licenses/by/4.0/. The Creative Commons Public Domain Dedication waiver (http://creativecommons.org/publicdomain/zero/1.0/) applies to the data made available in this article, unless otherwise stated in a credit line to the data. 


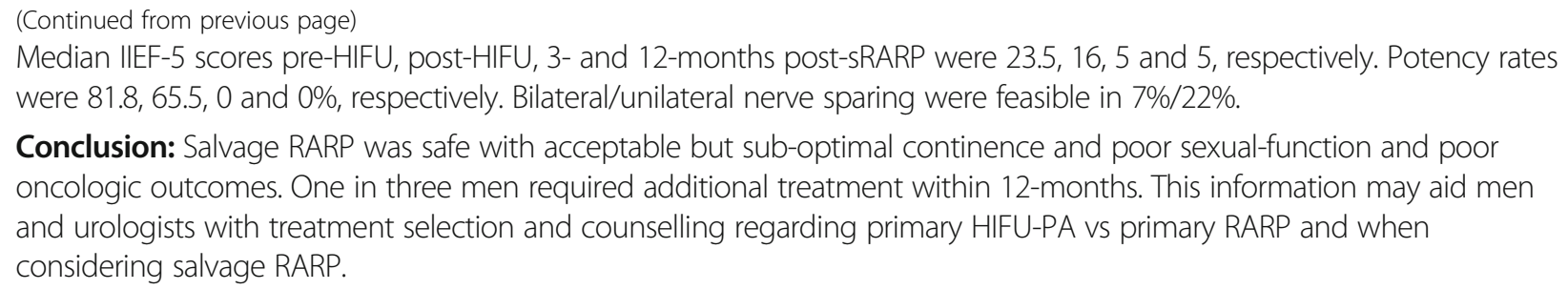

Keywords: Prostate cancer, HIFU, Salvage therapy, Radical prostatectomy, Robotics

\section{Background}

Partial Ablation (PA) is proposed as a less morbid alternative to radical therapy for localized prostate cancer [1]. High-intensity focused ultrasound (HIFU) is one of several ablative modalities that have been approved for clinical use within prospective registry studies in the UK [2], although it remains experimental according to international guidelines [3, 4]. Evidence of oncologic efficacy for HIFU-PA is limited to observational cohorts with short follow-up, using PSA or radical treatment-free survival endpoints and without comparison to a matched group undergoing radical treatment $[1,5,6]$.

Prostate cancer is often multi-focal and thus local recurrence after PA is common; $37-41 \%$ experienced recurrence requiring further treatment within 5 years and more than half $(54 \%)$ by 8 -years in two large, multicentre series $[6,7]$, despite the fact that only a third underwent biopsy after HIFU; the rate of recurrence may therefore increase with longer follow-up.

There is very little published data on outcomes for salvage RARP after HIFU-PA. sRARP after radiotherapy is associated with adverse pathologic features, high PSM rates, low continence rates, poor erectile function and higher anastomotic leak/stricture rates compared to primary RARP [8-10]. After PA, Nunes-Silva et al. reported similar continence but worse sexual function and higher recurrence rates for sRARP compared to primary RARP in a matched-pair analysis [11]. Marconi et al. recently reported on their cohort of 82 men undergoing sRARP after FA by two surgeons in which they demonstrated safety and feasibility as well as impressive $83 \%$ pad-free continence at 1 year; however, potency was low (14\% at 1 year) and the incidence of BCR was high (64\% at 3years) [12].

The most commonly used PA modality in the UK is HIFU and yet there are presently no published cohorts reporting on sRARP purely after HIFU-PA. This limits treatment selection and pre-treatment counselling for men with local recurrence after HIFU-PA or considering HIFU-PA as primary therapy.

We recently reported detailed pathologic outcomes in a series of 35 men and found high rates of multifocal cancer, Gleason upgrading, locally advanced ( $\mathrm{pT} 3 \mathrm{a} / \mathrm{b})$ cancer and positive margins, with a high rate of pT3 recurrence in-field and most PSMs being in-field due to the challenges of dissecting microscopic T3 cancer when it was densely fibrotic to the neurovascular bundles, pelvic floor muscles and rectum [13]. The primary objective of the present study was therefore to describe perioperative, oncologic and functional outcomes of sRARP following HIFU-PA.

\section{Methods \\ Study design and participants}

Shortly after commencing sRARP procedures for recurrence post-HIFU, the Department of Pelvic UroOncology at UCLH established a prospectively maintained electronic database to audit and analyse safety, oncologic and functional outcomes of sRARP postHIFU. Data is collected and entered in real-time by a team of clinical nurse specialists and research fellows, using a combination of medical records, pathology reports and validated questionnaires. This is the first comprehensive analysis reporting all of these outcomes from this database.

53 men underwent sRARP after focal therapy from January 2012-August 2017 at our institution. Data on the exact number of men who underwent focal HIFU up until 2017 was not available to the authors to ascertain a precise estimate of recurrence, but a recent study from two institutions (including ours) reported on outcomes for 1032 men who underwent focal HIFU over 20052017 [7]. Some of the men in that cohort study underwent salvage RARP at other institutions for which the results of sRARP are published elsewhere [12]; therefore the aforementioned cohort study is better able to assess the incidence of failure and salvage treatment than the present cohort.

We excluded 8 men, because including men with bilateral cancer (3 whole-gland HIFU) and ablative technologies that have dissimilar tissue effects ( 5 had other ablative modalities, i.e. cryotherapy or RFA) may otherwise have confounded outcomes. Median (IQR) followup was 17 months (IQR 15.4-20.5) after sRARP and 46.4 months (IQR 33.6-68.6) after HIFU. 33 men had $\geq 12$-months follow-up. 


\section{Eligibility/selection for HIFU}

All men had MRI and biopsy confirming prostate cancer and were deemed suitable for PA via MDT; most were 'ideal' candidates in line with prospective study protocols [14] or expert consensus criteria [15, 16]: PSA $\leq 15 \mathrm{ng} /$ $\mathrm{mL}$; ISUP grade group 1-3; cT2; uni-focal on MRI and biopsy; all were considered suitable for HIFU-PA at the time of treatment. On retrospective evaluation by the focal therapy team, however, 19/45 were not 'ideal' or received sub-optimal treatment according to (i) subsequently refined selection criteria or (ii) anatomic relative contra-indications (e.g. large gland, anterior/ apical location, capsular abutment suspicious for EPE) or (iii) technical treatment problems (e.g. probe problems, prominent calcifications, suboptimal Uchida changes suggesting incomplete ablation).

\section{Interventions and follow-up post-HIFU}

All HIFUs were performed as part of UK-wide registry or NCRI studies [2] using the Sonoblate $500^{\circ}$ device (Sonacare Medical, North Carolina). Our monitoring protocol following HIFU was described previously [14], including 3-monthly PSA, 6-12 month MRI and targeted/ template biopsy for abnormal MRI.

\section{Selection criteria for SRARP}

Given this is a retrospective analysis, no prospective selection criteria were defined. General selection criteria for sRARP were:

- Unsuitable for redo FA (e.g. bilateral/ high-risk cancer) or preference towards radical treatment;

- Age < 75yo and fit for major surgery;

- T1-3aNOM0, surgically resectable on MRI and DRE;

- Accepting of risks and side effects of surgery.

Pre-operative imaging included pre-biopsy MRI routinely, then bone scan and/or Ga68-PSMA PET CT for high-risk patients.

Salvage RARPs were performed by experienced surgeons ( $>250$ primary RARPs); biopsies and sRARP histology was reported by sub-specialist uro-pathologists using $5 \mathrm{~mm}$ axial step-sectioning with apical and basal shaves.

\section{Outcomes evaluated}

Clinical, imaging and pathologic characteristics (preHIFU, pre-sRARP, and post-sRARP) were collected. PSM rates were reported overall and stratified by pTstage, focality (single vs multifocal), length $(<3$ vs $\geq 3$ $\mathrm{mm}$ ) and relation to ablation zone (in-field versus outof-field). Clinically 'significant' margin was defined as pT3 PSM or $\geq 3 \mathrm{~mm}$ length or multifocal.

For post-sRARP oncologic outcomes: (i) Residual biochemical disease was defined as PSA $\geq 0.2 \mathrm{ng} / \mathrm{mL}$ and rising on successive measurements without postoperative PSA nadir $<0.2 \mathrm{ng} / \mathrm{ml}$;

(ii) $\mathrm{BCR}$ was defined as no residual biochemical disease followed by a PSA $\geq 0.2 \mathrm{ng} / \mathrm{mL}$ and rising on 2 successive measurements.

(iii) Adjuvant treatment was defined as initiation of $\mathrm{ADT} / \mathrm{RT}$ for high-risk pathologic features or residual biochemical disease;

(iv) Salvage treatment was defined as initiation of ADT/ RT for BCR.

Peri-operative parameters were extracted from operation reports and discharge summaries. Complications were attained from discharge summaries, clinic letters, emergency department records and urology nurse phone calls after sRARP. Complications were classified using the Clavien grading system as early ( $<90$ days) or late (90-365 days).

Continence and sexual function data were extracted from prospective questionnaires by independent researchers/nurse specialists. Pre- and post-HIFU-PA, leak-free and pad-free status were assessed via EPICQOL. After sRARP at 3- and 12-months, continence outcomes (number of pads/24h) were assessed via ICIQ-SF. Primary sexual function variables were IIEF-5 score (5-25) and Q2 of the EPIC/IIEF sexual function domain, i.e. "how often are erections firm enough for sexual intercourse" $(1-5: 1=$ none, $3=$ half, $5=$ all of the time). Q2 responses 3-5 were defined as potent for binary endpoint analysis. PDE5 inhibitors were permitted but men requiring invasive erectile aids were classified as impotent.

\section{Results}

CLINICO-PATHOLOGIC CHARACTERISTICS (pre-HIFU, preand post-sRARP)

Clinico-pathologic characteristics pre-HIFU, pre-sRARP and post-sRARP are shown in Table 1 including age, PSA, Gleason sum, cancer volume and cT-stage.

HIFU treatment details are shown in Table 2 including the proportion who were ideal in retrospect according to consensus selection criteria, type of biopsy pre-HIFU the anatomic extent of the ablation field in each patient, the number of treatments and presence of low-grade cancer left untreated.

Median time from diagnosis to sRARP was 43-months (IQR 30-67); median time from last HIFU to sRARP was 30-months (IQR 18-63).

\section{PERI-operative outcomes and complications}

Peri-operative outcomes and complications are shown in Table 3. Median (IQR) operative-console duration was 140 min (120-180). Median (IQR) blood loss was $200 \mathrm{ml}$ 
Table 1 - Comparative clinico-pathologic characteristics pre-HIFU, pre-sRARP and post-sRARP $(n=45)$

\begin{tabular}{|c|c|c|c|}
\hline Variables & Pre-HIFU & Pre-sRARP & Final sRARP pathology \\
\hline Age, years (IQR) & $62($ IQR 60-66) & 63.0 yrs. (IQR 61-66) & - \\
\hline PSA, median (IQR) & $7.3(5.5-8.9)$ & $6(3.9-10.0)$ & - \\
\hline \multicolumn{4}{|l|}{ Gleason score, n (\%): } \\
\hline $3+3$ & $9(20.0)$ & $3(6.7)$ & $0(0)$ \\
\hline $3+4$ & $29(64.4)$ & $22(48.9)$ & $22(48.9)$ \\
\hline $4+3$ (or $3+4$ with tertiary grade 5 ) & $5(11.1)$ & $15(33.3)$ & $18(40.0)$ \\
\hline $4+4$ (or $4+3$ with tertiary grade 5$)$ & $2(4.4)$ & $2(4.4)$ & $1(2.2)$ \\
\hline $4+5$ & - & $3(6.7)$ & $4(8.9)$ \\
\hline \multicolumn{4}{|l|}{ T-stage* ${ }^{*}$ n (\%): } \\
\hline $\mathrm{T} 2$ & $43(95.6)$ & $40(88.9)$ & $16(35.6)$ \\
\hline T3a & $2(4.4)$ & $4(8.9)$ & $21(46.7)$ \\
\hline T3b & - & $1(2.2)$ & $8(17.8)$ \\
\hline Max cancer core length in mm, median (IQR) & $7(5-10)$ & $6(3-8)$ & $\mathrm{n} / \mathrm{a}$ \\
\hline Number of cores taken, median (IQR) & $36(14-58)$ & $13(8-24)$ & $\mathrm{n} / \mathrm{a}$ \\
\hline Percentage of positive cores, median (IQR) & $17(11-30)$ & $30(14-40)$ & $\mathrm{n} / \mathrm{a}$ \\
\hline
\end{tabular}

(100-300). Median (IQR) length of stay was 1 day (1-2). The incidence of early (<90 days) Grade 1, 2 and 3 complications were $8.9,6.7$ and $2.2 \%$, respectively; the incidence of late (90-365 days) post-operative complications requiring re-admission was $15.2 \%$ (Table 3 ).

\section{Oncologic outcomes}

The overall (clinically significant) PSM rate was $44.4 \%$ (26.3\%), with $81 \%$ PSMs being in-field. The pT2 overall (clinically significant) margin rate was $37.5 \%$ (12.5\%), with $83.3 \%$ PSMs being in-field. The pT3

Table 2 - HIFU treatment technical details $(n=45)$

\begin{tabular}{|c|c|}
\hline Ideal according to consensus criteria, n (\%) & $26(57.8)$ \\
\hline Prostate volume, median (IQR) & $35(27-46)$ \\
\hline \multicolumn{2}{|l|}{ Location of treatment (combined treatment fields for $n=5$ with $2 \mathrm{HIFU}$ treatments) } \\
\hline \multicolumn{2}{|l|}{ Hemi-gland unilateral } \\
\hline Hemi-gland with extension across midline or into SV & 16 \\
\hline Hemi-gland anterior & 7 \\
\hline Hemi-gland posterior & 1 \\
\hline Quadrant (e.g. unilateral posterior) & 1 \\
\hline Focal ablation (eg posterior right basal segment) & 13 \\
\hline \multirow[t]{2}{*}{ Subtotal (extended hemi-ablation, sparing lateral aspect of contralateral side) } & 6 \\
\hline & 1 \\
\hline \multicolumn{2}{|l|}{ Number of HIFU treatments } \\
\hline 1 & 37 \\
\hline 2 & 8 \\
\hline \multicolumn{2}{|l|}{ Known 'insignificant' cancer left untreated at HIFU } \\
\hline Yes & $21(47.7)$ \\
\hline No & $23(52.3)$ \\
\hline \multicolumn{2}{|l|}{ Type of biopsy pre-HIFU: } \\
\hline TTMB TP 5 mm Mapping + MRI-Targeted (if targets) & $24(53.3)$ \\
\hline 12-20 core TRUS + MR-targeted (if targets) & $17(37.8)$ \\
\hline Targeted alone & $3(6.7)$ \\
\hline Not documented & $1(2.2)$ \\
\hline
\end{tabular}


Table 3 - Early and Late complications after sRARP according to Clavien group ( $n=45)$

\begin{tabular}{|c|c|c|}
\hline Early complications (< 90 days) & Number (\%) & Description \\
\hline \multirow[t]{2}{*}{ Grade I } & \multirow[t]{2}{*}{$4(8.9 \%)$} & (i) $1 \times$ AKI (self-limiting) \\
\hline & & (ii) 3 asymptomatic leaks on initial cystogram requiring prolonged catheterisation \\
\hline \multirow[t]{3}{*}{ Grade 2} & \multirow[t]{3}{*}{$3(6.7 \%)$} & (i) $1 \times$ UTI 2 weeks post-op requiring oral antibiotics; \\
\hline & & $\begin{array}{l}\text { (ii) } 1 \times \text { readmission for anastomotic leak and fever requiring IV antibiotics and } \\
\text { observation (no intervention) }\end{array}$ \\
\hline & & (ii) $1 x$ transfusion for retroperitoneal bleeding (did not require surgical/ radiologic intervention) \\
\hline $\begin{array}{l}\text { Grade 3a } \\
\text { Grade 3b }\end{array}$ & $1(2.2 \%)$ & $\begin{array}{l}\text { 3b: Laparotomy, evacuation of clot and re-fashinoing of vesico-urethral anastomosis for } \\
\text { haematoma causing anastomotic leak/ disruption }\end{array}$ \\
\hline Grade 4 & 0 & - \\
\hline Grade 5 (Death) & 0 & - \\
\hline Total & $8 / 45(17.8 \%)$ & - \\
\hline \multirow{3}{*}{$\begin{array}{l}\text { Late complications } \\
\text { (90 days }-12 \text { months)* }\end{array}$} & \multirow[t]{3}{*}{$5 / 33(15.2 \%)$} & (i) $3 x$ bladder neck contractures requiring 1 or more cystoscopy + optical dilation \\
\hline & & (ii) $1 \times$ Hemolock clip protruding into anastomosis causing LUTS \\
\hline & & $\begin{array}{l}\text { (iii) } 1 \times \text { Small bowel obstruction (resolved with conservative management) due to } \\
\text { adhesions in the same man who underwent laparotomy }<90 \text { days. }\end{array}$ \\
\hline
\end{tabular}

*Note to Table: All 45 men completed 90-day peri-operative outcome follow-up; 12 men have not yet reached 12-months follow-up and therefore the sample size is $n=33$

positive margin rate was $48.3 \%$, with $85.7 \%$ PSMs being in-field.

HIFU-PA did not appear to compromise oncologic outcomes in the out-of-field regions. The rate of out-offield (OOF) PSMs was very low, i.e. 6\% for pT2 (17\% OOF of $37.5 \%$ with PSM) and $7 \%$ for pT3 (14\% OOF of $48 \%$ with PSM). This suggests that the effects of HIFUPA on tissues away from the ablation zone were limited, such that surgeons were able to perform precise surgical dissection and also this suggests that tumour volumes may have been low in OOF regions, further reducing the probability of a PSM.

38 men had 3-month post-RP PSA data; $15.8 \%$ of men had residual biochemical disease (BCD) requiring immediate adjuvant ADT/RT; and additional 5.3\% (2/38) received early adjuvant $\mathrm{ADT}+\mathrm{RT}$ due to high risk features (both pT3a/b + ISUP 3-5 + PSM) and the remainder were managed expectantly; $10.5 \%$ (4/38) experienced $\mathrm{BCR}$ requiring salvage $\mathrm{ADT} / \mathrm{RT}$ (three within 12 months, one at 18 months). Of men with minimum 12month follow-up or having reached the ADT/RT event endpoint, $66.7 \%(22 / 33)$ were free from BCR and any adjuvant/salvage therapy at 12 months and 63.6\% (21/33) at last follow-up (median 18-months). No men experienced local/distant clinical recurrence or died during follow-up.

\section{Continence outcomes}

44/45 men had 3-month pad-free status documented ( $n=1$ with missing data due to failure to attend followup). 2 additional men were excluded from post-sRARP analysis due to using pads pre-sRARP for urge incontinence. At 3 (12) months post-sRARP, 33.3\% (65.5\%) were pad-free and $61.9 \%(86.2 \%)$ were socially continent, respectively. 3 \& 12-month median (IQR) ICIQ-scores were 4 (IQR 0-9) and 0 (0-3), respectively.

\section{Feasibility of nerve sparing at SRARP post-HIFU}

At sRARP, unilateral (bilateral) nerve sparing was feasible in $22.2 \%(6.7 \%) ; 11.1 \%$ of all NVBs (45 men $\times 2$ NVBs per patient) were preserved. Nerve sparing was not feasible due to HIFU-induced NVB fibrosis in $60.8 \%$, suspicion of posterolateral EPE on MRI or high-grade cancer near the NVB in $23.0 \%$ and erectile dysfunction in $17.9 \%$.

\section{Sexual function outcomes}

90.9\% (40/44) were potent pre-HIFU based on Q2 EPIC, IIEF-5 and/or clinical notes documentation of erectilefunction; $73.0 \%$ were potent post-HIFU. No man $(0 / 31)$ recovered erectile-function at 3 or 12 -months postsRARP

\section{PENTAFECTA outcomes in men with at least 12-months follow-up}

No man achieved the composite pentafecta [17] outcome, defined as all of: (i) potent adequate for intercourse half the time or more with/ without PDE5Is; (ii) continent not requiring any daily pad use; (iii) free from BCR; (iv) negative surgical margins; and (v) free from early or late peri-operative complications. 8/29 (27.6\%) achieved optimal outcomes across the four domains excluding potency. 


\section{Descriptive comparison to primary RARP outcomes}

Table 4 compares our published primary RARP outcomes $[18,19]$ to our salvage RARP outcomes detailed herein.

\section{Discussion}

To our knowledge, this is the second largest reported cohort of sRARP after PA and the first report on clinical outcomes purely using HIFU-PA as the ablative modality (Marconi et al. and Nunes-Silva et al. included a variety of PA ablative techniques). We found that sRARP is technically feasible, reasonably safe, and has acceptable continence outcomes long-term. However, erectilefunction recovery and oncologic results were poorer than expected for a cohort whom started out with favourable disease, and continence outcomes are arguably not ideal. These results are consistent with the recent report by Marconi et al [12], with the exception that in the present study the continence rate was lower (66\% vs $83 \%)$ and the significant PSM rate was higher (26\% vs $13 \%)$, possibly as a result of a greater proportion of focal re-treatments pre-sRARP in our series.

In this cohort study, sRARP had acceptable perioperative outcomes overall, but with higher than expected rates of anastomotic leaks and bladder neck contractures; this may be due to HIFU-induced tissue damage to the bladder neck and/or urethra in some cases. A recent report suggests that tissue-scaffold bio-grafts may help reduce the risk of leaks in salvage cases [20]. The apparently worse continence outcomes compared to primary RARP in our series, also seem unfavourable compared to the report from Nunes-Silva et al [11], possibly

Table 4 Summary of primary versus salvage RARP outcomes at our institution

\begin{tabular}{|c|c|c|}
\hline Baseline or Outcome Variable & Primary $\operatorname{RARP}(16,17)$ & Salvage RARP \\
\hline \multicolumn{3}{|l|}{ Complication rate (Clavien-Dindo grade) (\%) } \\
\hline Early Grade 1-3 & $7-13$ & 17.8 \\
\hline Early Grade 4 & $0.4^{*}$ & 0 \\
\hline Early Grade 5 & $0^{*}$ & 0 \\
\hline Anastomotic leak on cystogram & $2^{*}$ & 11.1 \\
\hline Late bladder neck contracture/ clip & $0.5^{*}$ & 10.5 \\
\hline \multicolumn{3}{|l|}{ Pre-RARP D’Amico risk group (\%) } \\
\hline Low & 3 & 6.7 \\
\hline Intermediate & 36 & 73.3 \\
\hline High & 61 & 20.0 \\
\hline \multicolumn{3}{|l|}{ Pathologic T-stage (\%) } \\
\hline pT2 & 53 & 35.5 \\
\hline pT3 & 47 & 64.5 \\
\hline рT3a & 33 & 46.6 \\
\hline pT3b & 14 & 17.8 \\
\hline \multicolumn{3}{|l|}{ Positive surgical margin rate (\%) } \\
\hline Overall & 17.3 & 44.4 \\
\hline pT2 & 9.6 & 37.5 \\
\hline pT3 & 26.1 & 48.3 \\
\hline \multicolumn{3}{|l|}{ Continence } \\
\hline Pad-free at 3-months & 67 & 33.3 \\
\hline Pad-free at 12-months & 85.4 & 65.5 \\
\hline Socially continent at 12-mo (0-1 pad) & 89.2 & 86.2 \\
\hline \multicolumn{3}{|l|}{ Proportion where nerve-sparing (NS) feasible } \\
\hline Feasibility of bilateral NS (\%) & 18 & 6.7 \\
\hline Feasibility of unilateral NS (\%) & 34 & 22.2 \\
\hline Feasibility of bilateral NS in high-risk Ca & 10 & 0.0 \\
\hline Proportion who received bilateral nerve-sparing and were potent at 12-months (potent pre-RARP)\# & 70 & $0(0 / 2)$ \\
\hline
\end{tabular}

*Institutional audit data from latest institutional audit for calendar year 2017, $n=605$ primary RARPs; \#defined as erections adequate for intercourse at least half the time with or without the aid of PDE5ls 
due to unmeasured differences in patient characteristics, extent of PA tissue damage or sRARP surgical technique. Further studies will provide a larger evidence base for continence outcomes of sRARP following HIFU, thus we are participating in a multi-institutional study (RAFT, NIH trial NCT03011606).

Sexual function outcomes in our series were poor, with bilateral nerve sparing rarely feasible due to a combination of post-HIFU fibrosis, cancer factors and erectile dysfunction. Potency preservation cannot thus be a realistic aim of sRARP. The PSM rate was high in this cohort (44.4\% overall, $37.5 \%$ pT2 and $48.3 \%$ pT3) despite wide excision at the site of previous HIFU and any highgrade or capsular tumor; this compares unfavorably to a recent report for primary RARP from our institution (17.3\% overall, $9.6 \%$ pT2 and $26.1 \%$ pT3). Likewise, in a meta-analysis of over 400,000 cases, the pT2 and pT3 PSM rates were 11 and 37\% [21]. PSM rates for sRARP after HIFU-PA were similar to salvage post-radiation [22], representing technical difficulty dissecting postHIFU tissue planes with severe fibrosis/adherence to the rectum and/or pelvic floor after HIFU.

Early oncologic outcomes were worse than expected for a selected population who at baseline mostly had unifocal, intermediate risk, cT2 disease. 1 in 3 men required further salvage therapy within 12 months. This is consistent with the matched-pair analysis of salvage versus primary RARP by Nunes-Silva et al. and the Marconi et al. cohort, which both reported high BCR rates in salvage cohorts. It might arguably be best to therefore perform wide bilateral excision in all sRARP cases after PA in an attempt to gain better oncologic control, especially as the potency recovery in these men is poor. The poor outcomes of sRARP vs primary RARP need to be balanced against the fact that in the short-term, propensity score-matched analysis suggest primary HIFU-PA has superior continence and potency outcomes to primary RARP, albeit with a significant risk of requiring salvage treatment [23, 24].

Our oncologic results were similar to sRARP following radiotherapy where one-third experience BCR within 12 months [25], half by 5 years [22] and almost two-thirds by 10 years [26]. It must be stressed, however, that these salvage RARP cohorts represent a minority of all primary HIFU cases and this study did not aim to estimate the incidence of failure after primary HIFU. As a guide, approximately 1000 focal HIFUs have been performed at UCLH between 2005 and 2017; 50 sRARPs were performed at UCLH, 20 elsewhere (via the RAFT study), 50 underwent radiotherapy at UCLH (others elsewhere), some received watchful waiting/ ADT and $~ 20 \%$ were re-treated with HIFU. Therefore, whilst quantifying the recurrence rate is difficult outside of prospective studies, we estimate $35-40 \%$, consistent with $37-41 \%$ reported by Guillaumier et al. and Stabile et al., in which $12 \%$ required radical treatment/ADT and another $25 \%$ received redo HIFU within 5 years [6], and $20 \%$ required radical therapy within 8 years [7].

Longer-term follow-up data is only available for whole gland HIFU; in two multi-centre registry study including cases from our institution, $37-41 \%$ required further therapy by 5 years and $54 \%$ by 8 years $[7,27]$, while another study reported $60 \%$ recurrence for intermediaterisk and $100 \%$ recurrence for high-risk disease by 10 years [28]. These studies suggest that the long-term (1015 year) risk of requiring radical therapy after HIFU may be higher than short-term studies suggest. Hence, we advise caution in patient selection and counselling until long-term follow-up data are available for men undergoing PA, especially for those at higher risk of recurrence e.g. ISUP group 3-5, younger age, family history, or Afro-Caribbean ethnicity. Our findings that the majority of recurrences were in-field and apical/ anterior suggest that other relative contra-indications may include apical or anterior tumours and large glands. Finally, any indication of sub-optimal ablation at the time of treatment (e.g. probe issues, anatomic issues, heavy calcification, reduced Uchida changes, inadequate treatment effects on post-HIFU MRI, etc. should warrant early re-biopsy and consideration of early salvage radical treatment.

This study has limitations. Data collection was prospective, but study design and analyses were retrospective. Non-standardized patient selection, pre-HIFU and post-HIFU biopsy techniques and selection for sRARP preclude from drawing conclusions about the efficacy of primary HIFU. Furthermore, this study cohort represents only a small subset of the overall HIFU cohort. Standardized validated questionnaires were used wherever possible to assess continence and erectile-function, but some men had missing baseline or follow-up questionnaires and thus binary endpoints using data from the medical case notes were relied upon. To minimize bias, we utilized only data that were collected independently by a third party (clinical nurse specialist or independent erectile dysfunction/incontinence clinic subspecialist urologist) in our analyses. Finally, although we made comparisons to our primary RARP cohort to provide context to our results, these were unadjusted for baseline variables. Hence, further studies with matchedpair analyses of salvage versus primary RARP are required to validate our findings.

Men who choose HIFU-PA as initial therapy for their localized prostate cancer do so to avoid the adverse effects of radical therapy. Given the recurrence rates of HIFU-PA, it is important these men are adequately counselled on outcomes of salvage radical therapy should their disease recur. For a proportion of men considering this novel therapy, the standard-care option of 
radical prostatectomy may become preferable given the data herein. Further, with recent innovations in RARP such as Retzius-sparing [29], which has been shown to decrease functional compromise, the incremental functional benefits of HIFU-PA over RARP may be reduced.

Standard-care options of management for locallyrecurrent prostate cancer in men after HIFU-PA are poorly defined, since HIFU-PA as initial therapy is not itself considered standard-care [3, 4]. Salvage options vary from systemic therapy to local radical therapy. sRARP is utilized by many high-volume expert RARP centres, and our data suggest that even in an experienced centre, overall outcomes are disappointing. Whether salvage radiotherapy provides better outcomes is unknown and is under investigation at our institution.

\section{Conclusions}

Salvage RARP for recurrent prostate cancer after HIFU$\mathrm{PA}$ is safe and feasible. Erectile recovery, continence recovery cancer recurrence and anastomotic stricture rates, however, appear worse than for primary RARP. HIFU-PA is an attractive choice to avoid the morbidity of radical treatment, but patients and their clinicians should consider the sub-optimal outcomes of sRARP when making initial and salvage treatment choices.

\section{Abbreviations}

ADT: Androgren Deprivation Therapy; BCR: Biochemical Recurrence; EPE: Extra-Prostatic Extension; HIFU-PA: High-Intensity Focussed Ultrasound Partial Ablation; ISUP: International Society of Uropathology; MRI: Magnetic Resonance Imaging; PCa: Prostate Cancer; PSM: Positive Surgical Margin; QOL: Quality of Life; RT: RadioTherapy; sRARP: Salvage Robotic-Assisted Radical Prostatectomy; UCLH: University College London Hospital

\section{Acknowledgements}

Nil

\section{Authors' contributions}

JT, AS, PS \& JK designed the study. JT, AS \& AM collected and checked the raw data. JT \& PS performed statistical analysis. GS, PR, PS, SN \& TB performed the surgeries. JT drafted the manuscript and compiled author edits, under supervision of JDK \& PS. GS, PR, TB, AS \& AM reviewed, edited and approved the final manuscript. The author(s) read and approved the final manuscript.

\section{Funding}

No funding was required for the study. All work done was on a voluntary basis and as part of the academic activities of the authors in their clinical appointments.

\section{Availability of data and materials}

De-identified raw datasets analysed for this study are available from the corresponding author on reasonable request, conditional on institutional data sharing agreements.

\section{Ethics approval and consent to participate}

The study was a retrospective analysis of de-identified data from a prospective institutional clinical audit database. No participant contact was required. The project was deemed low or negligible risk and the need for approval or consent was waived.

\section{Consent for publication}

(Not applicable).

\section{Competing interests}

The authors declare that they have no competing interests.

\section{Author details}

${ }^{1}$ Department of Uro-oncology, University College London Hospital, London W1G 8PH, UK. ${ }^{2}$ Faculty of Medicine, University of New South Wales, Kensington, Australia. ${ }^{3}$ Division of Surgery \& Interventional Sciences, University College London, London, UK. ${ }^{4}$ Centre for Molecular Oncology, Barts Cancer Institute, Queen Mary University of London, London, UK.

${ }^{5}$ Nuffield Department of Surgical Sciences, University of Oxford, Oxford, UK.

Received: 7 November 2019 Accepted: 22 June 2020

Published online: 01 July 2020

\section{References}

1. Veereman $G$, Jonckheer $P$, Desomer $A$, et al. Systematic review of the efficacy and safety of high-intensity Focussed ultrasound for localised prostate Cancer. Eur Urol Focus. 2015;1(2):158-70.

2. National Institute of Clinical Excellence UK. Focal therapy using highintensity focused ultrasound for localised prostate cancer. Vol Interventional Procedures Guidance (IPG) 424. United Kingdom2012.

3. Mottet N, Bellmunt J, Bolla M, et al. EAU-ESTRO-SIOG guidelines on prostate Cancer. Part 1: screening, diagnosis, and local treatment with curative intent. Eur Urol. 2017;71(4):618-29.

4. Sanda MG, Cadeddu JA, Kirkby E, et al. Clinically Localized Prostate Cancer: AUA/ASTRO/SUO Guideline. Part I: Risk Stratification, Shared Decision Making, and Care Options. The Journal of urology. 2017.

5. Warmuth M, Johansson T, Mad P. Systematic review of the efficacy and safety of high-intensity focussed ultrasound for the primary and salvage treatment of prostate cancer. Eur Urol. 2010;58(6):803-15.

6. Guillaumier S, Peters M, Arya M, et al. A multicentre study of 5-year outcomes following focal therapy in treating clinically significant nonmetastatic prostate Cancer. Eur Urol. 2018.

7. Stabile A, Orczyk C, Hosking-Jervis F, et al. Medium-term oncological outcomes in a large cohort of men treated with either focal or hemiablation using high-intensity focused ultrasonography for primary localized prostate cancer. BJU Int. 2019.

8. Takeda T, Tin AL, Corradi RB, et al. Topography of Prostate Cancer Recurrence After Radiation Therapy: A Detailed Mapping Study of Salvage Radical Prostatectomy Specimens. European urology. 2017.

9. Mandel P, Steuber T, Ahyai S, et al. Salvage radical prostatectomy for recurrent prostate cancer: verification of European Association of Urology guideline criteria. BJU Int. 2016;117(1):55-61.

10. Philippou Y, Parker RA, Volanis D, Gnanapragasam VJ. Comparative oncologic and toxicity outcomes of salvage radical prostatectomy versus nonsurgical therapies for Radiorecurrent prostate Cancer: a meta-regression analysis. Eur Urol Focus. 2016;2(2):158-71.

11. Nunes-Silva I, Barret E, Srougi V, et al. Effect of prior focal therapy on perioperative, oncologic and functional outcomes of salvage robotic assisted radical prostatectomy. J Urol. 2017;198(5):1069-76.

12. Marconi L, Stonier T, Tourinho-Barbosa R, et al. Robot-assisted radical prostatectomy after focal therapy: oncological, functional outcomes and predictors of recurrence. Eur Urol. 2019.

13. Thompson JE SA, Tan WS, Freeman A, Haider A, Allen C, Moore C, Orczyk C, Stabile A, Mazzon G, Rajan P, Shaw G, Mohammed A, Briggs TP, Nathan S, Sooriakumaran P, Kelly JD. Pathologic findings and MRI concordance at salvage radical prostatectomy for local recurrence following high-intensity focused ultrasound partial ablation. Journal of Urology. 2018;in press.

14. Dickinson L, Ahmed HU, Kirkham AP, et al. A multi-Centre prospective development study evaluating focal therapy using high intensity focused ultrasound for localised prostate cancer: the INDEX study. Contemporary clinical trials. 2013;36(1):68-80.

15. Tay KJ, Scheltema MJ, Ahmed HU, et al. Patient selection for prostate focal therapy in the era of active surveillance: an international Delphi consensus project. Prostate Cancer Prostatic Dis. 2017;20(3):294-9.

16. Donaldson IA, Alonzi R, Barratt D, et al. Focal therapy: patients, interventions, and outcomes--a report from a consensus meeting. Eur Urol. 2015;67(4): $771-7$.

17. Patel VR, Sivaraman A, Coelho RF, et al. Pentafecta: a new concept for reporting outcomes of robot-assisted laparoscopic radical prostatectomy. Eur Urol. 2011;59(5):702-7. 
18. Cathcart P, Sridhara A, Ramachandran N, Briggs T, Nathan S, Kelly J. Achieving quality Assurance of Prostate Cancer Surgery during Reorganisation of Cancer services. Eur Urol. 2015;68(1):22-9.

19. Sridhar AN, Cathcart PJ, Yap T, et al. Recovery of baseline erectile function in men following radical prostatectomy for high-risk prostate Cancer: a prospective analysis using validated measures. J Sex Med. 2016;13(3):435-43.

20. Ogaya-Pinies G, Kadakia Y, Palayapalayam-Ganapathi H, et al. Use of scaffolding tissue biografts to bolster Vesicourethral anastomosis during salvage robot-assisted prostatectomy reduces leak rates and catheter times. Eur Urol. 2018;74(1):92-8.

21. Tewari A, Sooriakumaran P, Bloch DA, Seshadri-Kreaden U, Hebert AE, Wiklund P. Positive surgical margin and perioperative complication rates of primary surgical treatments for prostate Cancer: a systematic review and meta-analysis comparing Retropubic, laparoscopic, and robotic prostatectomy. Eur Urol. 2012.

22. Chade DC, Eastham J, Graefen M, et al. Cancer control and functional outcomes of salvage radical prostatectomy for radiation-recurrent prostate cancer: a systematic review of the literature. Eur Urol. 2012;61(5):961-71.

23. Albisinni S, Aoun F, Bellucci S, et al. Comparing high-intensity focal ultrasound Hemiablation to robotic radical prostatectomy in the Management of Unilateral Prostate Cancer: a matched-pair analysis. Journal of endourology / Endourological Society. 2017;31(1):14-9.

24. Capogrosso P, Barret E, Sanchez-Salas R, et al. Oncological and functional outcomes of elderly men treated with HIFU vs. minimally invasive radical prostatectomy: a propensity score analysis. European journal of surgical oncology : the journal of the European Society of Surgical Oncology and the British Association of Surgical Oncology. 2018;44(1):185-91.

25. Bonet X, Ogaya-Pinies G, Woodlief T, et al. Nerve-sparing in salvage robotassisted prostatectomy: surgical technique, oncological and functional outcomes at a single high-volume institution. BJU Int. 2018.

26. Chade DC, Shariat SF, Cronin AM, et al. Salvage radical prostatectomy for radiation-recurrent prostate cancer: a multi-institutional collaboration. Eur Urol. 2011;60(2):205-10

27. Dickinson L, Arya M, Afzal N, et al. Medium-term outcomes after wholegland high-intensity focused ultrasound for the treatment of nonmetastatic prostate Cancer from a multicentre registry cohort. Eur Urol. 2016;70(4):66874.

28. Limani K, Aoun F, Holz S, Paesmans M, Peltier A, van Velthoven R. Single high intensity focused ultrasound session as a whole gland primary treatment for clinically localized prostate cancer: 10-year outcomes. Prostate Cancer. 2014;2014:186782.

29. Dalela D, Jeong W, Prasad MA, et al. A pragmatic randomized controlled trial examining the impact of the Retzius-sparing approach on early urinary continence recovery after robot-assisted radical prostatectomy. Eur Urol. 2017;72(5):677-85

\section{Publisher's Note}

Springer Nature remains neutral with regard to jurisdictional claims in published maps and institutional affiliations.

Ready to submit your research? Choose BMC and benefit from:

- fast, convenient online submission

- thorough peer review by experienced researchers in your field

- rapid publication on acceptance

- support for research data, including large and complex data types

- gold Open Access which fosters wider collaboration and increased citations

- maximum visibility for your research: over $100 \mathrm{M}$ website views per year

At $\mathrm{BMC}$, research is always in progress.

Learn more biomedcentral.com/submissions 